ISSN: 1311-1728 (printed version); ISSN: 1314-8060 (on-line version) doi: http://dx.doi.org/10.12732/ijam.v34i2.8

\title{
NUMERICAL APPROXIMATION METHOD FOR THE SPACE-DEPENDENT TELEGRAPH PROBLEM
}

\author{
Dounia Belakroum \\ Laboratory of Applied Mathematics \\ and Modelisations MAM, Department of Mathematics \\ Mentouri Brothers University Constantine - 25000 \\ ALGERIA
}

\begin{abstract}
In this paper we apply the Sinc-Galerkin method to construct a numerical solution of a telegraph equation with the variable coefficient. Several examples are provided to illustrate the accuracy and computational efficiency of the method are given.
\end{abstract}

AMS Subject Classification: 35L05, 42A10

Key Words: numerical solution; telegraph equation; Sinc-Galerkin method

\section{Introduction}

Mathematical modelling of physical systems in various fields of science leads often to linear and nonlinear partial differential equations. One such example is the telegraph equation, which is used in the study of wave propagation of electric signals in a cable transmission line and also in other wave phenomena. This equation has been extensively studied for initial and Dirichlet conditions by several methods for example see [2].

A lot of attention has been given in the literature to the Sinc-Galerkin method [1], [3], [4] and [5], its efficiency has been proved for both linear and nonlinear boundary value problems. The purpose of our present work is to introduce a numerical approach based on the Sinc function for the approximate

Received: December 15, 2020

(C) 2021 Academic Publications 
solution of a hyperbolic telegraph equation with the variable coefficient.

The outline of the remaining of the paper is as follows. In Section 2, we review the Sinc-Galerkin approach for the solution of the telegraph equation. Then our results are given in Section 3, where a new approximate numerical method to compute the proposed Sinc-Galerkin solution. We end with Section 4 of illustrative examples, where three problems are numerically solved with the proposed method. The results confirm the efficiency of the new scheme for solving the telegraph equation.

\section{Problem and Preliminaries}

Let us consider the following one-dimensional telegraph equation with the variable coefficient

$$
\frac{\partial^{2} u}{\partial t^{2}}+a \frac{\partial u}{\partial t}+b(x) u-\frac{\partial^{2} u}{\partial x^{2}}=f(x, t)
$$

for all $(x, t) \in(0,1) \times(0,+\infty)$.

Subject to the initial and boundary conditions

$$
u(x, 0)=u_{t}(x, 0)=0, u(0, t)=u(1, t)=0,
$$

where $a$ is positive constant, $b$ is continuous function on $[0,1]$.

We shall assume that $u$ and $f$ are analytic functions with respect to $x$ in a neighbourhood of $[0,1]$ and $t$ in $t \geq 0$.

\subsection{Sinc basis functions and quadrature}

Here we review the necessary notations, definitions and results associated with the Sinc-Galerkin method to solve problem (1). For more details [4] and [5] are given as excellent references. Then, our contributions are given in Section 3. Let $\mathbb{C}$ denote the set of all complex numbers.

Definition 1. For all $z \in \mathbb{C}$, define the Sinc function by

$$
\operatorname{sinc}(z)=\left\{\begin{array}{l}
\frac{\sin (\pi z)}{\pi z}, \quad z \neq 0 \\
1, \quad z=0 .
\end{array}\right.
$$

For $h>0$, the translated Sinc function with evenly spaced nodes is given for 
$k=0, \pm 1, \pm 2, \ldots$ by

$$
S(k, h)(z)=\operatorname{sinc}\left(\frac{z-k h}{h}\right)=\left\{\begin{array}{l}
\frac{\sin \left(\pi \frac{(z-k h)}{h}\right)}{\pi \frac{(z-k h)}{h}}, \quad z \neq k h, \\
1, \quad z=k h .
\end{array}\right.
$$

If $f(x)$ is defined on the real line, then for $h>0$ the Whittaker cardinal expansion of $f$ is given by

$$
f_{m}(x)=\sum_{k=-N}^{+N} f_{k} S(k, h)(x), m=2 N+1,
$$

where $f_{k}=f\left(x_{k}\right), x_{k}=h k$ and the mesh size is given by

$$
h=\sqrt{\frac{\pi d}{\alpha N}}, 0<\alpha \leq 1, d \leq \frac{\pi}{2},
$$

where $N$ is suitably chosen and $\alpha$ depends on the asymptotic behaviour of $f(x)$.

The $n$-th derivative of the function $f$ at the sampling points $x_{k}=k h$ can be approximated by using a finite number of terms as

$$
f^{(n)}(x) \approx h^{-n} \sum_{j=-N}^{N} \delta_{j k}^{(n)} f_{k},
$$

where

$$
\delta_{j k}^{(n)}=\frac{d^{n}}{d x^{n}}[S(j, h)(x)]_{x=x_{k}} .
$$

In particular

$$
\begin{aligned}
\delta_{j k}^{(0)}=\left.[S(j, h) o \phi(x)]\right|_{x=x_{k}}= \begin{cases}1, & k=j, \\
0, & k \neq j,\end{cases} \\
\delta_{j k}^{(1)}=\left.h \frac{d}{d \phi}[S(j, h) o \phi(x)]\right|_{x=x_{k}}= \begin{cases}0, & k=j, \\
\frac{(-1)^{k-j}}{(k-j)}, & k \neq j,\end{cases} \\
\delta_{j k}^{(2)}=\left.h^{2} \frac{d^{2}}{d \phi^{2}}[S(j, h) o \phi(x)]\right|_{x=x_{k}}= \begin{cases}\frac{-\pi^{2}}{3}, & k=j, \\
\frac{-2(-1)^{k-j}}{(k-j)^{2}}, & k \neq j .\end{cases}
\end{aligned}
$$


The interpolation formula for $f(x)$ over $[a, b]$ takes the form

$$
f(x) \approx \sum_{k=-N}^{+N} f_{k} S(k, h) o \phi(x)
$$

where the basis functions on $(a, b)$ are then given by

$$
S(k, h) o \phi(x)=\operatorname{sinc}\left(\frac{\phi(x)-k h}{h}\right),
$$

and the transformation function

$$
\phi(x)=\ln \left(\frac{x-a}{b-x}\right),
$$

transforms $[a, b]$ to the infinite range $[-\infty,+\infty]$.

The interpolation points $\left\{x_{k}\right\}$ are then given by $x_{k}=\frac{a+b e^{k h}}{1+e^{k h}}$.

The quadrature formula of $F(x)$ is given by

$$
\int_{a}^{b} F(x) d x \approx h \sum_{j=-N}^{N} \frac{F\left(x_{j}\right)}{\phi^{\prime}\left(x_{j}\right)} .
$$

\subsection{The Sinc-Galerkin Method}

We consider a Sinc approximation to the solution $u(x, t)$ of $(1)-(2)$ given by the formula

$$
u_{m_{x}, m_{t}}(x, t)=\sum_{i=-N_{x}}^{N_{x}} \sum_{j=-N_{t}}^{N_{t}} u_{i, j} S_{i, j}(x, t),
$$

where $m_{x}=2 N_{x}+1, m_{t}=2 N_{t}+1$ and

$$
S_{i, j}(x, t)=S_{i}(x) S_{j}(t)=\left[S\left(i, h_{x}\right) o \phi(x)\right]\left[S\left(j, h_{t}\right) o \psi(t)\right] .
$$

In this paper we take

$$
\phi(x)=\ln \left(\frac{x}{1-x}\right), \psi(t)=\ln (t) .
$$

Let us define the inner product of two functions $f$ and $g$ by

$$
<f, g>=\int_{0}^{\infty} \int_{0}^{1} f(x, t) g(x, t) W(x, t) d x d t
$$


where $W(x, t)$ is a weight function, conveniently taken to be

$$
W(x, t)=w(x) v(t)=\left[\frac{1}{\phi^{1 / 2}}\right]\left[\psi^{\prime}(t)\right]^{-1 / 2} .
$$

The unknown coefficients $u_{i, j}$ in (15) can be determined by the orthogonalization of the residual with respect to the Sinc basis functions $S_{i, j}(x, t)$. This yields the discrete Galerkin system

$$
<L u_{N_{x}, N_{t}}-f(x, t), S_{k l}>=0,-N_{x} \leq k \leq N_{x},-N_{t} \leq l \leq N_{t},
$$

where

$$
L u: u \longrightarrow \frac{\partial^{2} u}{\partial t^{2}}+a \frac{\partial u}{\partial t}+b(x) u-\frac{\partial^{2} u}{\partial x^{2}} .
$$

\section{Main Results}

Theorem 2. The following relations hold

$$
\begin{gathered}
<f(x, t), \mathcal{S}_{k} \mathcal{S}_{l}>\approx h_{t} h_{x} \frac{w\left(x_{k}\right) f\left(x_{k}, t_{l}\right) v\left(t_{l}\right)}{\phi^{\prime}\left(x_{k}\right) \psi^{\prime}\left(t_{l}\right)}, \\
<b(x) u, \mathcal{S}_{k} \mathcal{S}_{l}>\approx h_{t} h_{x} \frac{w\left(x_{k}\right) b\left(x_{k}\right) u\left(x_{k}, t_{l}\right) v\left(t_{l}\right)}{\phi^{\prime}\left(x_{k}\right) \psi^{\prime}\left(t_{l}\right)}, \\
<\frac{\partial^{2} u}{\partial x^{2}}, \mathcal{S}_{k} \mathcal{S}_{l}>\approx h_{t} h_{x} \frac{v\left(t_{l}\right)}{\psi^{\prime}\left(t_{l}\right)} \sum_{i=-N_{x}}^{N_{x}} \sum_{j=0}^{2} \frac{u\left(x_{i}, t_{l}\right)}{\phi^{\prime}\left(x_{i}\right)}\left[\frac{1}{h_{x}^{j}} \delta_{k i}^{(j)} \rho_{j}\right], \\
<\frac{\partial^{2} u}{\partial t^{2}}, \mathcal{S}_{k} \mathcal{S}_{l}>\approx \tau h_{t} h_{x} \frac{w\left(x_{k}\right)}{\phi^{\prime}\left(x_{k}\right)} \sum_{j=-N_{t}}^{N_{t}} \sum_{i=0}^{2} \frac{u\left(x_{k}, t_{j}\right)}{\psi^{\prime}\left(t_{j}\right)}\left[\frac{1}{h_{t}^{i}} \delta_{l j}^{(i)} \eta_{i}\right], \\
<a \frac{\partial u}{\partial t}, \mathcal{S}_{k} \mathcal{S}_{l}>\approx a h_{t} h_{x} \frac{w\left(x_{k}\right)}{\phi^{\prime}\left(x_{k}\right)} \sum_{j=-N_{t}}^{N_{t}} \sum_{i=0}^{1} \frac{u\left(x_{k}, t_{j}\right)}{\psi^{\prime}\left(t_{j}\right)}\left[\frac{1}{h_{t}^{i}} \delta_{l j}^{(i)} \mu_{i}\right]
\end{gathered}
$$

for some functions $\rho_{j}, \eta_{i}$, and $\mu_{i}$ to be determined.

Proof. One has

$$
<f(x, t), S_{k} S_{l}>=\int_{0}^{\infty} \int_{0}^{1} f(x, t) S_{k}(x) S_{l}(t) w(x) v(t) d x d t .
$$


Applying the Sinc quadrature rule (14) and using (10) yields

$$
\begin{aligned}
<f(x, t), S_{k} S_{l}> & \approx \int_{0}^{\infty} h_{x} \sum_{i=-N_{x}}^{N_{x}} \frac{f\left(x_{i}, t\right) S_{k}\left(x_{i}\right) S_{l}(t) w\left(x_{i}\right) v(t)}{\phi^{\prime}\left(x_{i}\right)} d t \\
& \approx h_{x} h_{t} \sum_{j=-N_{t}}^{N_{t}} \frac{f\left(x_{k}, t_{j}\right) S_{l}\left(t_{j}\right) w\left(x_{k}\right) v\left(t_{j}\right)}{\phi^{\prime}\left(x_{k}\right) \psi^{\prime}\left(t_{j}\right)} .
\end{aligned}
$$

Therefore,

$$
<f(x, t), S_{k} S_{l}>\approx h_{x} h_{t} \frac{f\left(x_{k}, t_{l}\right) w\left(x_{k}\right) v\left(t_{l}\right)}{\phi^{\prime}\left(x_{k}\right) \psi^{\prime}\left(t_{l}\right)} .
$$

In the same way, for the term $b(x) u$, the inner product with Sinc basis element gives

$$
\begin{aligned}
<b(x) u, S_{k} S_{l}> & =\int_{0}^{\infty} \int_{0}^{1} b(x) u(x, t) S_{k}(x) S_{l}(y) w(x) v(t) d x d t \\
& \approx h_{x} h_{t} \sum_{j=-N_{t}}^{N_{t}} \frac{b\left(x_{k}\right) u\left(x_{k}, t_{j}\right) S_{l}\left(t_{j}\right) w\left(x_{k}\right) v\left(t_{j}\right)}{\phi^{\prime}\left(x_{k}\right) \psi^{\prime}\left(t_{j}\right)}
\end{aligned}
$$

and consequently,

$$
<b(x) u, S_{k} S_{l}>\approx h_{x} h_{t} \frac{b\left(x_{k}\right) u\left(x_{k}, t_{l}\right) w\left(x_{k}\right) v\left(t_{l}\right)}{\phi^{\prime}\left(x_{k}\right) \psi^{\prime}\left(t_{l}\right)} .
$$

Moreover, we have

$$
<\frac{\partial^{2} u}{\partial x^{2}}, S_{k} S_{l}>=\int_{0}^{\infty}\left(\int_{0}^{1} \frac{\partial^{2} u(x, t)}{\partial x^{2}} S_{k}(x) S_{l}(t) w(x) v(t) d x\right) d t .
$$

As $u$ satisfies the boundary conditions (2) and due the fact that $w=0$ at $x=0,1$, doing integration by parts twice with respect to $x$, gives

$$
<\frac{\partial^{2} u}{\partial x^{2}}, \mathcal{S}_{k} \mathcal{S}_{l}>\approx h_{t} h_{x} \frac{v\left(t_{l}\right)}{\psi^{\prime}\left(t_{l}\right)} \sum_{i=-N_{x}}^{N_{x}} \frac{u\left(x_{i}, t_{l}\right)}{\phi^{\prime}\left(x_{i}\right)}\left(\mathcal{S}_{k}(x) w(x)\right)_{x=x_{i}}^{\prime \prime} .
$$

Using the relations (9)-(11), we get

$$
<\frac{\partial^{2} u}{\partial x^{2}}, \mathcal{S}_{k} \mathcal{S} l>\approx h_{t} h_{x} \frac{v\left(t_{l}\right)}{\psi^{\prime}\left(t_{l}\right)}
$$




$$
\begin{aligned}
& \times \sum_{i=-N_{x}}^{N_{x}} \frac{u\left(x_{i}, t_{l}\right)}{\phi^{\prime}\left(x_{i}\right)}\left[\frac{w\left(x_{i}\right) \phi^{\prime \prime}\left(x_{i}\right)}{h_{x}} \delta_{k i}^{(1)}+\frac{w\left(x_{i}\right) \phi^{\prime}\left(x_{i}\right)}{h_{x}^{2}} \delta_{k i}^{(2)}\right. \\
& \left.+2 \frac{w^{\prime}\left(x_{i}\right) \phi^{\prime}\left(x_{i}\right)}{h_{x}} \delta_{k i}^{(1)}+w^{\prime \prime}\left(x_{i}\right) \delta_{k i}^{(0)}\right] \\
& \approx h_{t} h_{x} \frac{v\left(t_{l}\right)}{\psi^{\prime}\left(t_{l}\right)} \sum_{i=-N_{x}}^{N_{x}} \frac{u\left(x_{i}, t_{l}\right)}{\phi^{\prime}\left(x_{i}\right)} \sum_{j=0}^{2}\left[\frac{1}{h_{x}^{j}} \delta_{k i}^{(j)} \rho_{j}\right],
\end{aligned}
$$

where

$$
\rho_{0}=w^{\prime \prime}, \quad \rho_{1}=\phi^{\prime \prime} w+2 \phi^{\prime} w^{\prime}, \quad \rho_{2}=\left(\phi^{\prime}\right)^{2} w .
$$

Similarly, for the term $\frac{\partial^{2} u}{\partial t^{2}}$, using integration by parts with respect to $t$ and noting that

$$
\lim _{t \rightarrow \infty} \frac{\partial u}{\partial t}(x, t) \mathcal{S}_{l}(t) v(t)=0, \quad \lim _{t \rightarrow \infty} u(x, t)\left(\mathcal{S}_{l}(t) v(t)\right)^{\prime}=0,
$$

and the conditions (2), we obtain

$$
\begin{aligned}
<\frac{\partial^{2} u}{\partial t^{2}}, \mathcal{S}_{k} \mathcal{S}_{l}> & =\int_{0}^{1} \mathcal{S}_{k}(x) w(x)\left(\int_{0}^{\infty} u(x, t)\left(\mathcal{S}_{l}(t) v(t)\right)^{\prime \prime} d t\right) d x \\
& \approx h_{t} h_{x} \frac{w\left(x_{k}\right)}{\phi^{\prime}\left(x_{k}\right)} \sum_{j=-N_{t}}^{N_{t}} \frac{u\left(x_{k}, t_{j}\right)}{\psi^{\prime}\left(t_{j}\right)}\left(\mathcal{S}_{l}\left(t_{j}\right) v\left(t_{j}\right)\right)^{\prime \prime} \\
& \approx h_{t} h_{x} \frac{w\left(x_{k}\right)}{\phi^{\prime}\left(x_{k}\right)} \\
& \times \sum_{j=-N_{t}}^{N_{t}} \frac{u\left(x_{k}, t_{j}\right)}{\psi^{\prime}\left(t_{j}\right)}\left(\mathcal{S}_{l}^{\prime \prime}\left(t_{j}\right) v\left(t_{j}\right)+2 \mathcal{S}_{l}^{\prime}\left(t_{j}\right) v^{\prime}\left(t_{j}\right)\right. \\
& \left.\quad+\mathcal{S}_{l}\left(t_{j}\right) v^{\prime \prime}\left(t_{j}\right)\right) .
\end{aligned}
$$

Thus, we can write

$$
<\frac{\partial^{2} u}{\partial t^{2}}, S_{k} S_{l}>\approx h_{t} h_{x} \frac{w\left(x_{k}\right)}{\phi^{\prime}\left(x_{k}\right)} \sum_{j=-N_{t}}^{N_{t}} \sum_{i=0}^{i=2} \frac{u\left(x_{k}, t_{j}\right)}{\psi^{\prime}\left(t_{j}\right)}\left[\frac{1}{h_{t}^{i}} \delta_{l j}^{(i)} \eta_{i}\right],
$$

where

$$
\eta_{0}=v^{\prime \prime}, \quad \eta_{1}=\psi^{\prime \prime} v+2 v^{\prime} \psi^{\prime}, \quad \eta_{2}=\left(\psi^{\prime}\right)^{2} v .
$$

Furthermore,

$$
<a \frac{\partial u}{\partial t}, \mathcal{S}_{k} \mathcal{S}_{l}>=\int_{0}^{1} \int_{0}^{\infty} a \frac{\partial u}{\partial t}(x, t) \mathcal{S}_{k}(x) \mathcal{S}_{l}(t) W(x, t) d t d x .
$$


Integrating by parts with respect to $t$ and due to $v=0$ at $t=\infty, u$ satisfies the initial conditions (2), the boundary term vanishes. Then, applying the quadrature rule, we get

$$
<a \frac{\partial u}{\partial t}, \mathcal{S}_{k} \mathcal{S}_{l}>\approx-a h_{t} h_{x} \frac{w\left(x_{k}\right)}{\phi^{\prime}\left(x_{k}\right)} \sum_{j=-N_{t}}^{N_{t}} \frac{u\left(x_{k}, t_{j}\right)}{\psi^{\prime}\left(t_{j}\right)}\left(\mathcal{S}_{l}\left(t_{j}\right) v\left(t_{j}\right)\right)^{\prime} .
$$

Using (9)-(11), we obtain

$$
<a \frac{\partial u}{\partial t}, \mathcal{S}_{k} \mathcal{S}_{l}>\approx-a h_{t} h_{x} \frac{w\left(x_{k}\right)}{\phi^{\prime}\left(x_{k}\right)} \sum_{j=-N_{t}}^{N_{t}} \frac{u\left(x_{k}, t_{j}\right)}{\psi^{\prime}\left(t_{j}\right)} \sum_{i=0}^{1}\left[\frac{1}{h_{t}^{i}} \delta_{l j}^{(i)} \mu_{i}\right],
$$

where

$$
\mu_{0}=v^{\prime}, \quad \mu_{1}=\psi^{\prime} v
$$

This completes the proof.

Using (16) with the approximations (17)-(21) in Theorem 2, we obtain that

$$
\begin{aligned}
& \frac{w\left(x_{k}\right)}{\phi^{\prime}\left(x_{k}\right)} \sum_{j=-N_{t}}^{N_{t}} \frac{u\left(x_{k}, t_{j}\right)}{\psi^{\prime}\left(t_{j}\right)} \sum_{i=0}^{2}\left[\frac{1}{h_{t}^{i}} \delta_{l j}^{(i)} \eta_{i}\right] \\
& -a \frac{w\left(x_{k}\right)}{\phi^{\prime}\left(x_{k}\right)} \sum_{j=-N_{t}}^{N_{t}} \frac{u\left(x_{k}, t_{j}\right)}{\psi^{\prime}\left(t_{j}\right)} \sum_{i=0}^{1}\left[\frac{1}{h_{t}^{2}} \delta_{l j}^{(i)} \mu_{i}\right]+\frac{w\left(x_{k}\right) b\left(x_{k}\right) u\left(x_{k}, t_{l}\right) v\left(t_{l}\right)}{\phi^{\prime}\left(x_{k}\right) \psi^{\prime}\left(t_{l}\right)} \\
& -\frac{v\left(t_{l}\right)}{\psi^{\prime}\left(l_{l}\right)} \sum_{i=-N_{x}}^{N_{x}} \frac{u\left(x_{i}, t_{l}\right)}{\phi^{\prime}\left(x_{i}\right)} \sum_{j=0}^{2}\left[\frac{1}{h_{t}^{j}} \delta_{k i}^{(j)} \rho_{j}\right]=\frac{w\left(x_{k}\right) f\left(x_{k}, t_{l}\right) v\left(t_{l}\right)}{\phi^{\prime}\left(x_{k}\right) \psi^{\prime}\left(t_{l}\right)}
\end{aligned}
$$

and we get the discrete Sinc-Galerkin system for the determination of unknown coefficients $\left\{u_{i, j},-N_{x} \leq i \leq N_{s},-N_{t} \leq j \leq N_{t}\right\}$. In order to write it in a matrix-vector form, we introduce the following notations:

Let $D\left(y_{x}\right)$ denote an $m_{x} \times m_{x}$ diagonal matrix whose diagonal elements are $y\left(x_{-N_{x}}\right), y\left(x_{-N_{x}+1}\right), \ldots, y\left(x_{N_{x}}\right) . I_{m_{x}}^{(p)}, p=0,1,2$ be the $m_{x} \times m_{x}$ matrices $I^{(p)}$ whose $j k$-th entry $\delta_{j k}^{(P)}$ is given by equations (9)-(11). Also the matrices $D\left(y_{t}\right), I_{m_{t}}^{(p)}, p=0,1,2$ are similarly defined though of size $m_{t} \times m_{t}$.

With these notations, the discrete system (25) can be written

$$
\begin{aligned}
& D\left(\frac{1}{\phi^{\prime}}\right) D(w) U D(v)\left[\sum_{j=0}^{2} \frac{1}{h_{t}^{j}} I_{m t}^{(j)} D\left(\frac{\eta_{j}}{\psi^{\prime} v}\right)\right]^{t} \\
& -a D\left(\frac{1}{\phi^{\prime}}\right) D(w) U D(v)\left[\sum_{j=0}^{1} \frac{1}{h_{t}^{j}} I_{m_{t}}^{(j)} D\left(\frac{\mu_{j}}{\psi^{\prime} v}\right)\right]^{t} \\
& -\left[\sum_{i=0}^{2} \frac{1}{h_{x}^{2}} I_{m_{x}}^{(i)} D\left(\frac{\rho_{i}}{\left(\phi^{\prime 2} w\right.}\right)\right] D\left(\phi^{\prime}\right) D(w) U D\left(\frac{v}{\psi^{\prime}}\right) \\
& +D(b) D\left(\frac{w}{\phi^{\prime}}\right) U\left(\frac{v}{\psi^{\prime}}\right)=D\left(\frac{w}{\phi^{\prime}}\right) F D\left(\frac{v}{\psi^{\prime}}\right),
\end{aligned}
$$


where []$^{t}$, denotes the transpose of the matrix [ ].

Multiplying system (26) at first by $D\left(\phi^{\prime}\right)$ and secondly multiplying it by $D\left(\psi^{\prime}\right)$ yields the equivalent system

$$
A X+X B=C,
$$

where

$$
\begin{aligned}
& A=A 1-a A 2, \quad B=-B 1+B 2, \\
& A 1=\left[\sum_{j=0}^{2} \frac{1}{h_{t}^{j}} I_{m_{t}}^{(j)} D\left(\frac{\eta_{j}}{\psi^{\prime} v}\right)\right]^{t} D\left(\psi^{\prime}\right), \\
& A 2=\left[\sum_{j=0}^{1} \frac{1}{h_{t}^{3}} I_{m_{t}}^{(j)} D\left(\frac{\mu_{j}}{\psi^{\prime} v}\right)\right]^{t} D\left(\psi^{\prime}\right), \\
& B 1=D\left(\phi^{\prime}\right)\left[\sum_{i=0}^{2} \frac{1}{h_{x}^{i}} I_{m_{x}}^{(i)} D\left(\frac{\rho_{i}}{\left(\phi^{\prime 2} w\right.}\right)\right] D\left(\phi^{\prime}\right), \\
& B 2=D(b), \quad C=D(w) F D(v)
\end{aligned}
$$

and

$$
X=D(w) U D(v)
$$

$A, B, X$ and $C$ are $m_{t} \times m_{t}, m_{x} \times m_{x}, m_{x} \times m_{t}$, and $m_{x} \times m_{t}$ matrices, respectively. $U$ and $F$ are $m_{x} \times m_{t}$ matrices whose $k l$-th entries are given by $u_{k l}$ and $f\left(x_{k}, t_{l}\right)=f\left(\frac{e^{k h}}{1+e^{k h}}, e^{l h}\right)$, respectively.

We obtain the $U_{i j}$ coefficients for the approximate Sinc-Galerkin solution by solving (27) for $X$.

\section{Applications}

In this section, we illustrate our numerical method on three problems. Our computations and graphical representations were done using the computer algebra system Maple. In all of the examples, we have used the absolute error, defined as

$$
\text { Absolute error }=\mid u_{\text {exact solution }}-u \text { Sinc-Galerkin solution } \mid
$$

with the choices

$$
N_{x}=N_{t}=N ; h_{x}=h_{t}=\frac{2}{\sqrt{N}}, x_{k}=\frac{e^{k h}}{1+e^{k h}}, t_{j}=e^{j s} .
$$




\subsection{Example 1}

In this example, we consider the following problem

$$
\left\{\begin{array}{l}
\frac{\partial^{2}}{\partial t^{2}} u(x, t)-\frac{\partial^{2}}{\partial x^{2}} u(x, t)=f(x, t), 0<x<1, t>0 \\
u(0, t)=u(1, t)=0, t \geq 0 \\
u(x, 0)=u_{t}(x, 0)=0,0 \leq x \leq 1
\end{array}\right.
$$

where

$$
f(x, t)=e^{-t}\left(2-4 t+t^{2}\right)\left(1-\cos ^{2}(\pi x)\right)-2 t^{2} e^{-t} \pi^{2}\left(2 \cos ^{2}(\pi x)-1\right) .
$$

The exact solution of this problem is given by

$$
u(x, t)=t^{2} e^{-t} \sin ^{2}(\pi x) .
$$

The graphics of the exact and approximate solution of problem (30), by using the Sinc-Galerkin method proposed in Section 3 are presented in Figure 1.

\begin{tabular}{ccc}
\hline$x$ & Absolute- error for $N=4$ & Absolute- error for $N=12$ \\
\hline 0.1 & $2.7047355421 \times 10^{-3}$ & $4.5425855434 \times 10^{-5}$ \\
0.4 & $8.300180448 \times 10^{-3}$ & $1.79140209911 \times 10^{-5}$ \\
0.7 & $4.576589504 \times 10^{-3}$ & $3.48358060752 \times 10^{-5}$ \\
\hline
\end{tabular}

Table 1: Absolute errors at $t=2.95$ for $N=4$ and $N=12$.

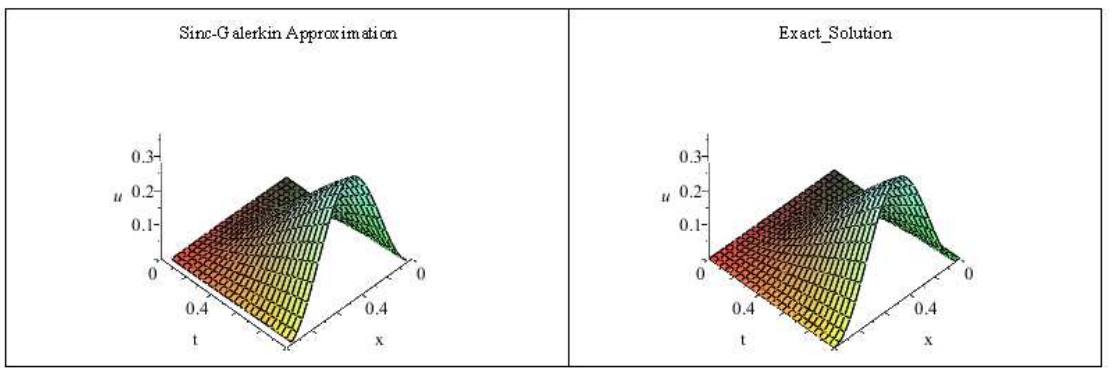

Figure 1: Exact and approximate solutions.

Values of absolute error (28) of the approximate solution to problem (30) are given at $t=2.95$ for different $N$ in Table 1 . 


\subsection{Example 2}

Now we consider the following telegraph problem

$$
\left\{\begin{array}{l}
\frac{\partial^{2}}{\partial t^{2}} u(x, t)+\frac{\partial}{\partial t} u(x, t)+\sqrt{x} u(x, t) \\
=\frac{\partial^{2}}{\partial x^{2}} u(x, t)+f(x, t), 0<x<1, t>0 \\
u(0, t)=u(1, t)=0, t \geq 0 \\
u(x, 0)=u_{t}(x, 0)=0,0 \leq x \leq 1
\end{array}\right.
$$

where

$$
\begin{aligned}
& f(x, t)=(1-x)^{5 / 2} t e^{-t}\left(6 x^{5 / 2}-3 t x^{5 / 2}+x^{3} t^{2}\right) \\
& +t^{3} e^{-t} x^{1 / 2}(1-x)^{1 / 2}\left(-\frac{15}{4}(1-x)^{2}+\frac{25}{2} x(1-x)-\frac{15}{4} x^{2}(1-x)\right) .
\end{aligned}
$$

The exact solution is given by

$$
u(x, t)=t^{3} e^{-t} x^{5 / 2}(1-x)^{5 / 2} .
$$

We get the approximated solution shown in Figure 2. Values of associated absolute errors at $t=1.64$ for $N=6$ and for $N=14$ are given in Table 2 .

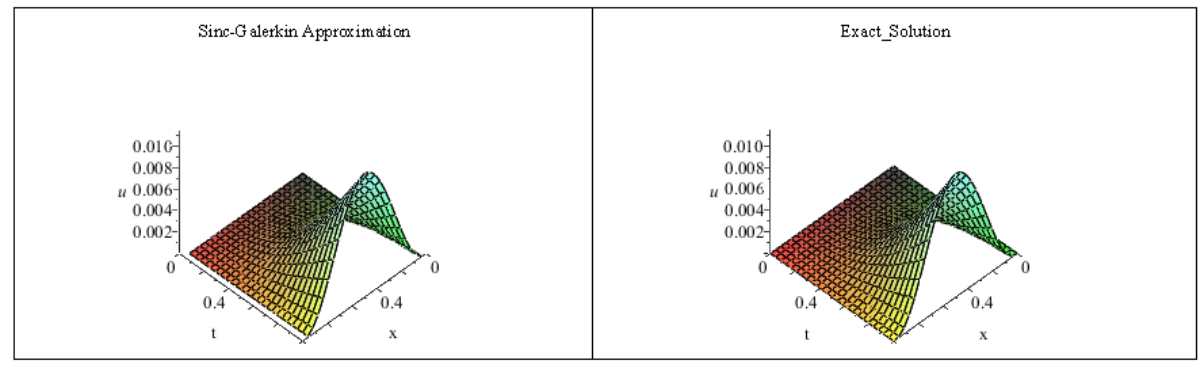

Figure 2: Exact and approximate solutions.

\subsection{Example 3}

Finally, in this example we consider

$$
\left\{\begin{array}{l}
\frac{\partial^{2}}{\partial t^{2}} u(x, t)+\frac{1}{25} \frac{\partial}{\partial t} u(x, t)+\left(e^{x}-1\right) u(x, t) \\
=\frac{\partial^{2}}{\partial x^{2}} u(x, t)+f(x, t), 0<x<1, t>0 \\
u(0, t)=u(1, t)=0, t \geq 0 \\
u(x, 0)=u_{t}(x, 0)=0,0 \leq x \leq 1
\end{array}\right.
$$




\begin{tabular}{ccc}
\hline $\mathrm{x}$ & Absolute-error for $N=6$ & Absolute-error for $N=14$ \\
\hline 0.10 & $4.68392505519 \times 10^{-4}$ & $2.27913727553 \times 10^{-5}$ \\
0.35 & $2.31773467117 \times 10^{-3}$ & $8.60182612193 \times 10^{-5}$ \\
0.60 & $2.58186034508 \times 10^{-3}$ & $9.34662132387 \times 10^{-5}$ \\
0.85 & $7.82807851173 \times 10^{-4}$ & $3.59828849007 \times 10^{-5}$ \\
\hline
\end{tabular}

Table 2: Absolute error at $t=1.64$ for $N=6$ and $N=14$.

where

$$
\begin{aligned}
& f(x, t)=x e^{x-t}\left(-2-4 x+2 x^{2}+t\left(8 x-4 x^{2}-4\right)+t^{2}\left(1-2 x+x^{2}\right)\right) \\
& +\frac{1}{25} t^{2} e^{x-t}\left(1-3 x+x^{2}+x^{3}\right)+\left(e^{x}-1\right) x(1-x)^{2} t^{2} e^{x-t} \\
& +t^{2} e^{x-t}\left(x^{3}+4 x^{2}-x-2\right) .
\end{aligned}
$$

The exact solution to this problem is

$$
u(x, t)=x(1-x)^{2} t^{2} e^{x-t} .
$$

Figure 3 plots the exact and the approximated solutions obtained by using the Sinc-Galerkin method with the choices (29). The absolute error is displayed in Table 3 for different $N$ at $t=2.89$

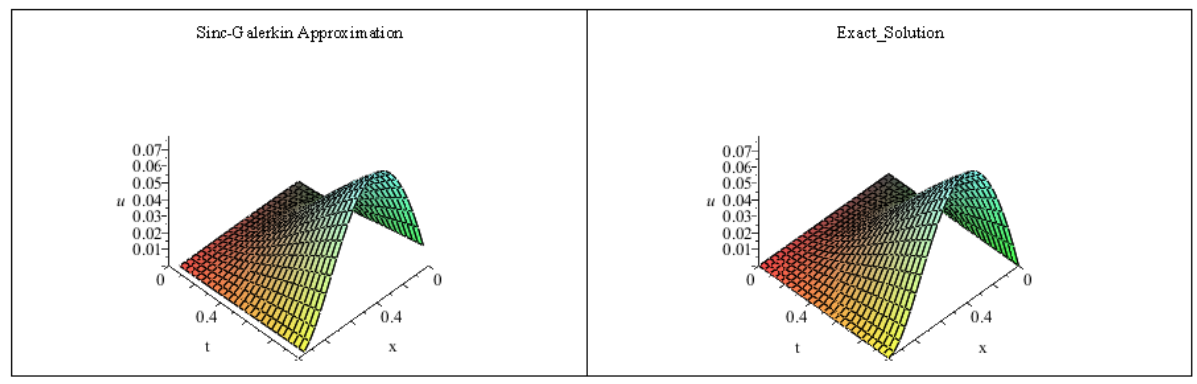

Figure 3: Exact and approximate solutions.

\section{Conclusion}

In this study, a numerical method based on Sinc functions is presented for solving telegraph type equation with variable coefficient. The numerical results obtained by the proposed method demonstrate the great accuracy. The author wishes that the procedure as described will considerably be for the benefit of solving any other partial differential equations. 


\begin{tabular}{ccc}
\hline $\mathrm{x}$ & Absolute-error for $N=6$ & Absolute-error for $N=15$ \\
\hline 0.10 & $2.15382501119 \times 10^{-3}$ & $5.180329637 \times 10^{-5}$ \\
0.35 & $2.08928466534 \times 10^{-3}$ & $4.43659273983 \times 10^{-5}$ \\
0.60 & $1.74457377693 \times 10^{-3}$ & $3.61434074388 \times 10^{-4}$ \\
0.85 & $7.68331978348 \times 10^{-4}$ & $2.88096656516 \times 10^{-4}$ \\
\hline
\end{tabular}

Table 3: Absolute error at $t=2.89$ for $N=6$ and $N=15$

\section{References}

[1] M. El-Gamel, Error analysis of Sinc-Galerkin method for time-dependent partial differential equations, Numer. Algorithms, 77, No 2 (2018), 517533.

[2] A. Guezane-Lakoud, D. Belakroum, Rothe's method for telegraph equation with integral conditions, Nonlinear Analysis, 70, (2009), 3842-3853.

[3] M. Kelly McArthur, K. L. Bowers and John Lund, Numerical implementation of the Sinc-Galerkin method for second-order hyperbolic equations, Numer. Methods Partial Differential Equations, 3, No 3 (1987), 169-185.

[4] J. Lund, K. L. Bowers, Sinc Methods for Quadrature and Differential Equations, SIAM, Philadelphia (1992).

[5] F. Stenger, Numerical Methods Based on Sinc and Analytic Functions, Springer-Verlag, New York (1993). 
